\title{
Factores de riesgo asociados a la obesidad infantil en niños atendidos en el Hospital I Tingo María Essalud - 2009.
}

\author{
Risk factors associates to the infantile obesity in children catered in the Hospital I Tingo Maria Essalud \\ $-2009$.
}

Marco Jesús Donayre Legua ${ }^{1}$

\section{RESUMEN}

Objetivo: Determinar los factores de riesgo asociados a la obesidad infantil en niños atendidos en el Hospital I Tingo María Essalud - 2009.Materiales y métodos: Se realizó un estudio retrospectivo, de casos y controles. Se estudió 51 casos de niños con obesidad infantil y 102 controles niños sin obesidad infantil, atendidos en el Hospital I Tingo María - ESSALUD en el año 2002, quienes fueron seleccionados de acuerdo a criterios de inclusión y exclusión. Se aplicó una encuesta estructurada como instrumento de recolección de datos. Resultados: La obesidad en los niños es una entidad patológica frecuente en población asegurada adscrita al Hospital I Tingo María ESSALUD. Los factores que presentan asociación estadística significativa con la obesidad infantil son el ingreso económico de los padres, el entorno obesigenico que rodea al niño y el sedentarismo o falta de actividad física en los niños, siendo sus O.R. respectivamente 2. 011 - 2,516 - 2,661.Conclusión: El factor que presenta el mayor nivel de asociación estadística con la obesidad infantil es el sedentarismo o la falta de actividad física, siendo su O. R. de 2,661. A nivel de políticas de salud es imprescindible generar condiciones de vida saludables aptas para el desarrollo pleno de los niños, implementando, fortaleciendo y reforzando las medidas de promoción, prevención y control de los factores asociados a la obesidad infantil, con énfasis en la actividad física.

Palabras Clave: Obesidad Infantil - Factores asociados

\begin{abstract}
Objective: To determine risk factors associated with childhood obesity in children treated at the Hospital I Tingo Maria Essalud - 2009. Materials and methods: Retrospective study of cases and controls. We studied 51 cases of children with childhood obesity and 102 controls without childhood obesity children treated at the Hospital I Tingo Maria - ESSALUD in 2002, who were selected according to inclusion and exclusion criteria. A structured questionnaire was applied as a tool for data collection. Results: The obesity in the children is a frequent pathological organization in assured population assigned to the Hospital I Tingo Maria ESSALUD. The factors that have statistically significant association with childhood obesity in the bivariate analysis are three, these being the income of the parents, the environment surrounding the child obesigenico and sedentary lifestyle or lack of physical activity in children, and its OR 2,011 respectively - 2.516 to 2.661.Conclusion: The factor that presents/displays the greater level of statistical association with the infantile obesity is the sedentarismo or the lack of physical activity, being his Or. R. of 2,661. At level of health policies it is essential to generate healthful conditions of life apt for the total development of the children, implementing, fortifying and reinforcing the measures of promotion, prevention and control of the factors associated to the infantile obesity, with emphasis in the physical activity.
\end{abstract}

Key words: Infantile obesity - Factors associated

\footnotetext{
${ }^{1}$ Médico Pediatra. Hospital I Tingo María, EsSalud, Huánuco, Perú.
} 


\section{INTRODUCCIÓN}

La obesidad infantil constituye un problema creciente de salud pública a nivel mundial, como consecuencia de los cambios en el estilo de vida que han modificado los hábitos alimentarios, con un consecuente aumento en el consumo de calorías y grasas y una disminución en la actividad física (1). Se afirma que más de la tercera parte de las consultas de endocrinología pediátrica corresponden a esta causa (2). El incremento alarmante de la frecuencia de obesidad infantil es un serio problema de salud, por asociarse a enfermedades crónicas en la edad adulta, como son: las enfermedades cardiovasculares, la diabetes mellitus (DM) y las dislipidemias, entre otras $(3,4,5)$. Estudios realizados en niños y adolescentes indican que el proceso de aterosclerosis comienza en la infancia y se asocia de forma significativa con la obesidad, así como con otros componentes del síndrome metabólico $(6,7)$. La prevalencia de la obesidad ha variado notablemente en relación con los últimos 20-30 años, y se estima que el porcentaje de niños y adolescentes obesos ha sufrido un dramático incremento de casi un $50 \%$ (8).

La importancia del presente proyecto de investigación se justifica por el incremento de la magnitud del problema, siendo la prevalencia de la obesidad infantil en niños menores de 15 años en población asegurada adscrita al Hospital I Tingo María en el año 2009 de 13\% (30), es importante resaltar que la población asegurada de menores de 15 años es la más afectada, debido probablemente a la condición de asegurado que corresponde a un estrato socioeconómico medio a alto y al estilo de vida poco vinculado a la actividad física y a una alimentación adecuada. Por lo explicitado la ejecución del proyecto nos permitirá conocer los factores más importantes que vienen ocasionando la obesidad infantil en nuestro medio y de esta manera contribuir a partir de la evidencia científica generada por el estudio, a implementar actividades de intervención costo efectivas para la prevención y control de la obesidad infantil en niños asegurados adscritos al Hospital I Tingo María ESSALUD.

El problema formulado fue:

¿Cuáles con los factores de riesgo asociados a la obesidad infantil en niños atendidos en el Hospital I Tingo María Essalud - 2009?

El objetivo general del estudio, determinar los factores de riesgo asociados a la obesidad infantil en niños atendidos en el Hospital I Tingo María
Essalud - 2009; y los objetivos específicos: Caracterizar en tiempo, espacio y persona la obesidad infantil en niños atendidos en el Hospital I Tingo María Essalud. Establecer si el ingreso económico, el entorno obesigénico y el sedentarismo son factores de riesgo asociados a la obesidad infantil y definir si el entorno obesigénico es el factor de riesgo más importante asociado a la obesidad infantil en niños atendidos en el Hospital I Tingo María.

\section{METODOLOGÍA}

El estudio fue, por el nivel de la investigación analítico/explicativo, por el tiempo de recolección de los datos retrospectivo, por el tipo de intervención del investigador observacional y por el tipo de diseño casos y controles.

La población en estudio estuvo conformada por el total de casos de obesidad infantil $(n=51)$ atendidos en la Cartera del Niño/adolescente del Hospital I Tingo María ESSALUD, en el año 2009. La conformación de los casos y controles se hizo de acuerdo a los criterios de inclusión y exclusión, aceptándose la relación de 1: 2 de caso / control.

El cálculo de tamaño muestral para el grupo de casos y controles no fue pertinente, el grupo de casos estuvo conformado por el total de casos de niños con obesidad infantil $(n=51)$; el grupo de controles estuvo conformado por niños normales según IMC correspondiente a la edad y el sexo, se acepta una relación de 1: 2 de caso / control, por lo tanto los controles serán ( $n=102)$, los controles fueron asignados al grupo por muestreo aleatorio simple.

Criterios de inclusión y exclusión: Para evitar los sesgos en la recolección de la información se utilizó criterios de inclusión y exclusión para seleccionar las unidades muestrales, a incluirse en el estudio.

Criterios de inclusión

Casos: Niños asegurados de ambos sexos, edad menor de 15 años, atendidos en el Hospital I Tingo María ESSALUD en el año 2009 y cuyo diagnóstico dado en el consultorio de Pediatría del Hospital sea obesidad infantil.

Controles: Niños asegurados de ambos sexos, edad menor de 15 años, atendidos en el Hospital I Tingo María ESSALUD en el año 2009 y cuyo diagnóstico dado en el consultorio de Pediatría del Hospital sea un niño normal. 
Criterios de exclusión

Casos: Niños asegurados de ambos sexos, edad menores de 15 años, atendidos en el Hospital I Tingo María ESSALUD en el año 2009 y cuyo diagnóstico dado en el consultorio de Pediatría del Hospital sea obesidad infantil y que son portadores de diabetes mellitus, desnutrición grave, neoplasias, quimioterapia o radioterapias, SIDA y otros cuadros crónicos degenerativos sistémicos.

Controles: Niños asegurados de ambos sexos, edad menores de 15 años, atendidos en el Hospital I Tingo María ESSALUD en el año 2009 y cuyo diagnóstico dado en el consultorio de Pediatría del Hospital sea niño normal y que no son portadores de diabetes mellitus, desnutrición grave, neoplasias, quimioterapia o radioterapias, SIDA y otros cuadros crónicos degenerativos sistémicos. Técnicas de recolección y procesamiento de datos Cuestionario: Para la recolección de datos de los niños con y sin obesidad se utilizó un cuestionario estructurado, instrumento que fue aplicado a cada madre del niño asegurado incluido en el estudio, a atención se realizó en el Hospital I Tingo María en el primer semestre del año 2009. La validación realizar el análisis estadístico bivariado para la comprobación de la hipótesis. del instrumento de recolección de datos fue por juicio de expertos (03 Médicos Especialistas Pediatras) Procesamiento de datos: Para el procesamiento de los datos se utilizo el software estadístico SPSS versión 15.0 para Windows y para el análisis de los datos y medir el nivel de asociación de las variables en estudio, el análisis univariado y bivariado, prueba del odds ratio, aceptándose como significativo valores $\mathrm{p}<0.05$.

\section{RESULTADOS}

La presentación de los resultados de la investigación por el tipo de diseño metodológico de caso / control y la búsqueda de la fuerza de asociaciones entre la variable dependiente (Obesidad Infantil) y la independiente (Factores de Riesgo Asociados), se realizó sobre la base de la ficha ad hoc utilizada en el estudio, que nos brinda información pertinente de las variables más relevantes asociadas a esta entidad nosológica, permitiendo de esta manera realizar inferencias válidas para el estudio. En primera instancia se realiza un análisis descriptivo de las variables más relevantes del estudio, para más adelante

La tabla 1, nos muestra que el ingreso económico alto de los padres de los niños, es un factor asociado a la obesidad infantil, esta variable presenta un OR $>1=2,011$.

Tabla 1: Relación entre el ingreso económico de los padres y la obesidad infantil en niños atendidos en el Hospital I Tingo María ESSALUD - 2009

\begin{tabular}{|c|c|c|c|c|}
\hline \multirow{2}{*}{$\begin{array}{c}\text { Ingreso económico } \\
\text { de los padres }\end{array}$} & \multicolumn{2}{|c|}{ Obesidad Infantil } & \multirow[b]{2}{*}{$\mathbf{P}$} & \multirow[b]{2}{*}{ OR } \\
\hline & NO & SI & & \\
\hline Alto & 54 & 38 & 0,098 & 2,011 \\
\hline Medio & 48 & 13 & & \\
\hline Bajo & & & & \\
\hline
\end{tabular}

La tabla 2, nos muestra que el entorno obesigenico o la presencia de dos o más personas con obesidad en el hogar del niño, es un factor asociado a la obesidad infantil, esta variable presenta un OR > $1=2.516$

Tabla 2: Relación entre el entorno obesigenico y la obesidad infantil en niños atendidos en el Hospital I Tingo María ESSALUD - 2009

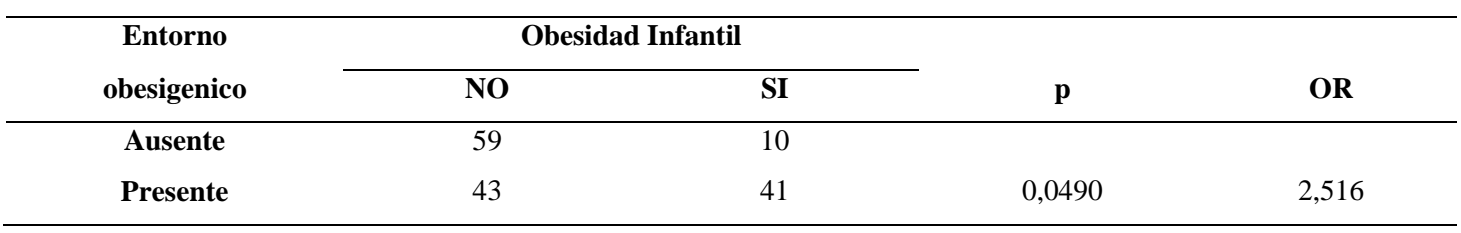


La tabla 3, nos muestra que el sedentarismo o la carencia de actividad física por parte de los niños incluidos en el estudio, es un factor asociado a la obesidad infantil, esta variable presenta un OR $>1=2,661$

Tabla 3: Relación entre el sedentarismo y la obesidad infantil en niños atendidos en el Hospital I Tingo María ESSALUD - 2009

\begin{tabular}{|c|c|c|c|c|}
\hline \multirow[t]{2}{*}{ Sedentarismo } & \multicolumn{2}{|c|}{ Obesidad Infantil } & \multirow[b]{2}{*}{$\mathbf{p}$} & \multirow[b]{2}{*}{ OR } \\
\hline & NO & SI & & \\
\hline Ausente & 55 & 8 & & \\
\hline Presente & 47 & 43 & 0,026 & 2,661 \\
\hline
\end{tabular}

En la Tabla 4, podemos apreciar de las seis variables más relevantes en estudio, tres son las que presentan asociación estadística significativa con la obesidad infantil, siendo estas el ingreso económico de los padres, el entorno obesigenico que rodea al niño y el sedentarismo o falta de actividad física en los niños, siendo sus O.R. respectivamente 2,011 - 2,516 - 2,661.

Tabla 4: Factores asociados a la obesidad infantil en niños atendidos en el Hospital I Tingo María ESSALUD - 2009

\begin{tabular}{|c|c|c|c|c|c|}
\hline \multirow{3}{*}{ Factores } & \multirow{3}{*}{ Categorías } & \multicolumn{2}{|c|}{ Obesidad Infantil } & \multirow{3}{*}{$\mathbf{p}$} & \multirow{3}{*}{ OR } \\
\hline & & NO & SI & & \\
\hline & & $(n=102)$ & $(n=51)$ & & \\
\hline \multirow[t]{3}{*}{ Edad } & $1-4$ & 50 & 20 & & \\
\hline & $5-9$ & 38 & 18 & & \\
\hline & $10-15$ & 14 & 13 & 0,751 & \\
\hline \multirow[t]{2}{*}{ Sexo } & Masculino & 56 & 22 & & \\
\hline & Femenino & 46 & 29 & 0,243 & \\
\hline \multirow[t]{3}{*}{ Procedencia } & Tingo María & 74 & 42 & & \\
\hline & Rural & 24 & 6 & & \\
\hline & Otros & 04 & 3 & 0,176 & \\
\hline Grado de Instrucción de & Superior & 37 & 42 & & \\
\hline \multirow[t]{3}{*}{ los padres } & Secundaria & 17 & 9 & & \\
\hline & Primaria & 05 & 8 & 0,767 & \\
\hline & Analfabeto & & & & \\
\hline Ingreso económico de los & Alto & 54 & 38 & & \\
\hline \multirow[t]{2}{*}{ padres } & Medio & 48 & 13 & $0,098 *$ & 2,011 \\
\hline & Bajo & & & & \\
\hline \multirow[t]{2}{*}{ Entorno obesigenico } & Ausente & 59 & 10 & & \\
\hline & Presente & 43 & 41 & $0,049 * *$ & 2,516 \\
\hline \multirow[t]{2}{*}{ Sedentarismo } & Ausente & 55 & 8 & & \\
\hline & Presente & 47 & 43 & $0,026 * *$ & 2,661 \\
\hline
\end{tabular}

Significativo con un nivel de confianza del 95\%

** Significativo con un nivel de confianza de $90 \%$

\section{DISCUSIÓN}

El primer año de vida, es el período de crecimiento y desarrollo más rápido en la vida del niño y es cuando este es más inmaduro y vulnerable. Debe recordarse que el lactante es especialmente sensible $y$ vulnerable ante transgresiones dietéticas y sus consecuencias son más severas que en el niño mayor y el adulto. Es aceptado ya, que los hábitos alimentarios se relacionan con muchas enfermedades como la obesidad, ateromatosis y cáncer, entre otras afecciones, por lo cual es importante la creación de hábitos correctos de alimentación en el niño y su familia 
para contribuir a la prevención de estos problemas de salud. Una anticipación en la introducción de alimentos diferentes a la leche materna pudiera provocar una ingesta de energía por encima de la recomendada y la creación de hábitos de alimentación inadecuados como la adicción al exceso de azúcares simples, fomentando la creación de hábitos alimentarios pre mórbidos para la obesidad (37).

Por tanto, no se deben introducir alimentos distintos de la leche materna antes de los seis meses de edad por factores propios del individuo dados por su madurez, crecimiento y desarrollo; por factores propios de los alimentos que no reúnan las características adecuadas para esta etapa de la vida y por las influencias negativas que tales precocidades pueden crear en la conducta alimentaria desde estas edades que pueden facilitar la obesidad $(38,39,40)$.

En este marco el presente estudio encontró del total de las variables consideradas para su contrastación, a tres variables que presentan asociación estadística significativa con la obesidad infantil, siendo estas el ingreso económico de los padres, el entorno obesigenico que rodea al niño y el sedentarismo o falta de actividad física en los niños, siendo sus O.R. respectivamente 2,011 - 2,516 - 2,661, este hallazgo concuerda con numerosos estudios que respaldan la asociación entre exceso de peso y una disminución de la actividad física, aumentando considerablemente el riesgo de resistencia insulínica, diabetes mellitas tipo II, enfermedad cardiovascular, hipertensión arterial e hiperlipidemias $(41,42,43)$. Otro estudio ha mostrado que la obesidad en el padre es un fuerte factor de riesgo para la obesidad infantil. La obesidad en los padres es un factor de riesgo bien conocido sobre la obesidad infantil. Si uno de los padres es obeso, la probabilidad de que el niño también lo sea se incrementa por tres, pero sí lo son ambos padres, el riesgo se multiplica por diez (44), evidencias que concuerdan con nuestro hallazgo respecto a los factores sedentarismo entendida para el estudio como falta de actividad física y entorno obesigenico comprendido como madre y/o padre con el problema de obesidad.

La asociación entre obesidad, actividad física y entorno obesigenico, entendido como padres con obesidad, es fácil de explicar y refuerza los resultados de otros estudios nacionales y extranjeros, sin embargo debo de manifestar que respecto al factor ingreso económico medio de los padres, la evidencia científica disponible obtenida permitió establecer que los escolares consumen una dieta poco saludable caracterizada por un bajo consumo de lácteos, frutas y verduras, siendo el factor ingreso económico bajo el que influye negativamente en el consumo de frutas y verduras, por el alto costo y las preferencias se inclinan a alimentos más ricos en grasas y azúcares debido probablemente factores culturales, los mismos que están asociados a obesidad infantil, lo cual es coincidente con lo encontrado por otros autores (45, 46); no obstante los resultados son discordantes con los hallazgos del presente estudio, cuyos resultados nos muestran que existe asociación entre la obesidad infantil y el ingreso económico medio, por tanto debemos de expresar que nuestros hallazgos son válidos para el escenario donde se llevó a cabo el estudio, siendo necesario llevar a cabo otros estudios que puedan definir el nivel de asociación de estas dos variables.

En el marco de lo expuesto podríamos concluir que la obesidad infantil ya es un problema de salud pública en la Región, de ello se desprende la necesidad de planificar intervenciones dirigidas a contrarrestar los factores de riesgo presentes en este grupo, basados en la estrategia implementada por el Ministerio de Salud "Intervención a través de Ciclos de Vida”, es posible intervenir con acciones concretas tales como, aumento de la actividad física moderada en las instituciones educativas de nivel inicial y primaria, educación alimentaria, entre otras, orientado a reducir la prevalencia de obesidad en los grupos de mayor riesgo.

Conclusiones:

- La obesidad en los niños es una entidad patológica frecuente en población asegurada adscrita al Hospital I Tingo María ESSALUD.

- Los factores que presentan asociación estadística significativa con la obesidad infantil son el ingreso económico de los padres, el entorno obesigenico que rodea al niño y el sedentarismo o falta de actividad física en los niños, siendo sus O.R. respectivamente 2. 011 - 2,516 - 2,661.

- El factor que presenta el mayor nivel de asociación estadística con la obesidad infantil es el sedentarismo o la falta de actividad física, siendo su O. R. de 2,661.

- Los factores como edad, sexo, procedencia y grado de instrucción de los padres, no presentan asociación con la obesidad infantil en esta zona. 


\section{AUTOR DE CORRESPONDENCIA:}

Marco Jesús Donayre Legua.

Hospital I Tingo María Essalud.

Jirón Bolognesi N ${ }^{\circ} 120$ - Tingo María, Rupa

Rupa, Leoncio Prado, Huánuco - Perú.

Teléfono: +51-1-962547790.

E-mail: donayrelema@yahoo.es

\section{REFERENCIAS BIBLIOGRAFICAS}

1. Gotthelf S, Jubany L. Prevalencia de factores de riesgo asociados al síndrome metabólico en niños y adolescentes obesos de la Ciudad de Salta, 2004.

2. Güell R, Carvajal M. Obesidad en el niño y el adolescente. En: De la Torre, González J, Gutiérrez JA, Jordán J, Pelayo EJ, eds. Pediatría. Tomo 7. La Habana: Editorial Ciencias Médicas; 2004.p.97-107.

3. Chiang $M$, Torres $M$, Maldonado $M$, González U. Propuesta de un programa de promoción sobre un estilo de vida saludable en preescolares mediante una intervención multidisciplinaria. Rev Cubana Invest Biomed. 2003;22:245-52.

4. Eisenmann J. Methodology on the use of a continuous metabolic syndrome score in pediatric research. Cardiovascular Diabetology. 2008;7:17.

5. Burrows R. Avances en el síndrome metabólico en niños con sobrepeso. J Pediatr. 2008;152:185-90.

6. Iannuzzi A, Licenziati MR, Acampora C. Carotidartery stiffness in obese children with the metabolic syndrome. Am J Cardiol. 2006;97:528-31.

7. Daniels SR, Arnett DK, Eckel RH. Overweight in children and adolescents pathophysiology, consequences, prevention, and treatment. Circulation. 2005;111:1999-2012.

8. Fagot-Campagna A. Emergence of type 2 diabetes mellitus in children: epidemiological evidence. $\mathrm{J}$ Pediatr Endocrinol Metab. 2000;13:1395-402.

9. Módulo Obesidad Infantil. Salud del Niño y del Adolescente, Salud Familiar y Comunitaria. D R A F T. Organización Panamericana de la Salud. 2002.

10. McLellan F.Obesity rising to alarming levels around the world. Lancet.2002; 359 (9315):1412.

11. Blair SN,KohlHWill Et Al: Physical fitnes and cause mortality: A prospective Study of Healthy men and womas Jma 262: 2395-2401.2003.
12. Quiñones Carmen, Ramalle Enrique, Perucha Milagros, Cruz Eugenia, et al. Factores de riesgo asociados con la presencia de obesidad en niños menores de 15 años. 2007.

13.PAJUELO, Jaime, ROCCA, Jesús y GAMARRA, Marco. Obesidad infantil: sus características antropométricas y bioquímicas. An. Fac. med., ene./mar. 2003, vol.64, no.1, p.21-26.

14. Hernández Bernardo, Cuevas Lucía, Teresa Shamah-Levy Teresa, Et al. "Factores asociados con sobrepeso y obesidad en niños mexicanos de edad escolar: resultados de la Encuesta Nacional de Nutrición”. Salud Publica Mex 2003;45 supl 4:S551-S557.

15.Johandra Argote Parolis; Tamara Fernández Terue; Francisco Carvajal Martínez; Roberto Manuel González Suárez; Manuel E. Licea Puig. "Prevalencia y factores asociados al síndrome metabólico en niños y adolescentes obesos del municipio Marianao”. 2007. Infomed.sld.cu

16. Brown G. Alberts JJ. Fissher LD et al. Regression of coronary artery disease as a result of intensive lipid- lowering therapy in men high levels of apolipoprotein B N Engl J Med. 323:1289 - 1298, 1990.

17. Harrison. Principios de Medicina Interna. 14 edición. McGraw- Hill Interamericana de España. 2000 pp 515- 528

18. Lancher et al , Diabetes Care Volumen 25 Number 4 April 2002 pp. 792 - 3

19. Holme l: Análisis de estudios rabdomizados que evalúan el efecto de la reducción del colesterol en la mortalidad total y la incidencia de enfermedad coronaria. Circulation 82:1916- 1624, 1990

20. Allison DB, Fontaine KR, Manson JE,et al. Annual deaths attributable to obesity in the United States JAMA 1999;282:1530.

21. Davidson MB. Clinical implications of insulin resistance syndromes. Am J Med 1995; 99 (4): 420-6.

22. Mc Teman CL, Mc Ternan PG, Harte AL, et al. Resistin, central obesity, ans type 2 diabetes. Lancet. 2002;359 (9300): 46-47.

23. Kathryn Señor, Resistina entre Obesidad y DM tipo 2. Vol. 357 enero 20, 2002 pp202

24. Bray G. Problemas de salud asociado a Obesidad. Uptodate 9.3 Jul. 30. 2001

25. Farreras. Tratado de Medicina Interna. Edición 2000. Evaluación de Obesidad. pág. 351-358. 
26. Gidding SS, Leible RI et al. Comprendiendo la Obesidad en Jóvenes. circulation 94: 3383- 3387

27. Bray G. Fisiopatologia de la obesidad. American Journal of clinical nutrition. Siplemento. 2000. Vol. 55 488s - 494s.

28. Epidemiologia Hospital I Tingo María. Reporte perfiles epidemiológicos. 2009.

29. Rojas, María Isabel. Instituto Especializado de Salud del Niño. Reporte niños con obesidad y sobrepeso, Servicio de Endocrinología 2007.

30. Salvatierra Ruiz Rosa. Centro Nacional de Nutrición y Alimentación (Cenan) del Instituto Nacional de Salud. INS. Perú. 2008.

31. Gavino, A. Problemas de alimentación en el niño. Manual práctico para su prevención y tratamiento de la obesidad. Editorial Pirámide, Madrid. 1998.

32. Saldaña, C. Trastornos del comportamiento alimentario. Madrid: Fundación Universidad-Empresa. 1994.

33. Bogado, Carlos. Falta de actividad física, sedentarismo. Asociación Patagonia Saludable. Revista Noticias Salud. Chile. Artículo publicado 19 Agosto 2009. www.noticiasnet.com.ar

34. Gerardo Moncada y Luisa Gómez Pombo. Fundación Heinrich Boell, Oxfam y Rostros y Voces FDS A.C. México, D.F. 2007.

35. Dei Cas, P. Overweight and obesity in 2 to 9 year old children: relationship with influencing factors. Arch Argent Pediatr 2002;100(5):368373
36. Monteiro PO, Victora CG. Rapid growth in infancy and childhood and obesity in later life a systematic review. Obes Rev. 2005;6(2):14354.

37. Knip M, Akerblom HK. Early nutrition and later diabetes risk. Adv Exp Med Biol. 2005;569:14250.

38. Koletzko B, Broekaert I, Demmelmair H, Franke J, Hannibal I, Oberle D, Schiess S, Baumann BT, Verwied Jorky S; EU Childhood Obesity Project. Protein intake in the first year of life: a risk factor for later obesity? The E.U. childhood obesity project. Adv Exp Med Biol. 2005;569:6979.

39. Albala C, Vio F, Kain J, Uauy R: Nutrition transition in Chile: determinants and consequences. Public Health Nutrition 2002; 5: 123-8. [ [ Links ]

40. Uauy R, Albala C, Kain C: Obesity Trends in Latin America: Transiting from Underto Overweight. INTA, Universidad de Chile. American Society for Nutritional Sciences, 2001. [ [ Links ]

41. Albala C, Vio F, Kain J, Uauy R: Nutrition Transition in Latin America: The Case of Chile. Nutr Rev 2001; 59: 1706. [ Links $]$

42. Committee on Nutrition. Prevention of PediatricOverweight and Obesity. Pediatrics 2003; 112(2):424-430.

43. Atalah E, Urteaga C, Rebolledo A, et al: Prevalencia de Obesidad en Escolares de la Región de Aysén. Rev Chil Pediatr 1999; 70: 208-14 [ Links ]

44. Araya M, Atalah E: Factores que determinan la selección de alimentos en familias de sectores populares. Rev Chil Nutr 2002; 29: 308-15. [ [ Links ]

Recibido: $01 / 07 / 2015$

Aceptado: 30 /09/2015 